\title{
Chronic kidney disease in propionic acidemia
}

\author{
Oleg A. Shchelochkov, MD ${ }^{1}$, Irini Manoli, MD, PhD ${ }^{1}{ }^{1}$, Jennifer L. Sloan, PhD, MS ${ }^{1}$, \\ Susan Ferry, BSN ${ }^{1}$, Alexandra Pass, BA ${ }^{1}$, Carol Van Ryzin, $\mathrm{MS}^{1}$, Jennifer Myles, $\mathrm{MS}^{2}$, \\ Megan Schoenfeld, BS ${ }^{2}$, Peter McGuire, MS, MD ${ }^{1}$, Douglas R. Rosing, MD', Mark D. Levin, MD ${ }^{3}$, \\ Jeffrey B. Kopp, $\mathrm{MD} \mathbb{1}^{4}$ and Charles P. Venditti, MD, $\mathrm{PhD}{ }^{1}{ }^{1}$
}

Purpose: Propionic acidemia (PA) is a severe metabolic disorder characterized by multiorgan pathology, including renal disease. The prevalence of chronic kidney disease (CKD) in PA patients and factors associated with CKD in PA are not known.

Methods: Thirty-one subjects diagnosed with PA underwent laboratory and clinical evaluations through a dedicated natural history study at the National Institutes of Health (ClinicalTrials.gov identifier: NCT02890342).

Results: Cross-sectional analysis of the creatinine-based estimated glomerular filtration rate (eGFR) in subjects with native kidneys revealed an age-dependent decline in renal function $(P<0.002)$. Among adults with PA, 4/8 (50\%) had eGFR $<60 \mathrm{~mL} / \mathrm{min} / 1.73 \mathrm{~m}^{2}$. There was a significant discrepancy between eGFRs calculated using estimating equations based on serum creatinine compared with serum cystatin $\mathrm{C}(P<0.0001)$. The tubular injury marker, plasma lipocalin-2, and plasma uric acid were strongly associated with CKD $(P<0.0001)$. The measured 24 -hour creatinine excretion was below normal, even after adjusting for age, height, and sex.

Conclusion: $\mathrm{CKD}$ is common in adults with PA and is associated with age. The poor predictive performance of standard eGFR estimating equations, likely due to reduced creatine synthesis in kidney and liver, could delay the recognition of CKD and management of ensuing complications in this population.

Genetics in Medicine (2019) 21:2830-2835; https://doi.org/10.1038/s41436019-0593-z

Keywords: propionic acidemia; chronic kidney disease; GFR; cystatin C; creatinine

\section{INTRODUCTION}

Propionic acidemia (PA) is a metabolic disorder that carries a marked risk for multiorgan pathology. ${ }^{1}$ Chronic kidney disease $(\mathrm{CKD}$, here narrowly defined as the glomerular filtration rate $<60 \mathrm{~mL} / \mathrm{min} / 1.73 \mathrm{~m}^{2}$ ) has been recognized with increased frequency as a complication in patients with organic acidemias, especially methylmalonic acidemia (MMA), and more recently, in other categorically related disorders such as $\mathrm{PA}$ and glutaric aciduria type $\mathrm{I}^{2-7}$ In $\mathrm{PA}$, the prevalence, natural history, and factors associated with CKD are incompletely defined.

Studies focused on renal function in PA are scant. An association between PA and CKD was first suggested in 1997 by Lehnert et al. ${ }^{6}$ Subsequent PA patient case reports of renal failure in a 45 -year-old woman with $\mathrm{CKD}^{5}$ and another who presented with stage $3 \mathrm{CKD}$ in the third decade of life ${ }^{7}$ have been bolstered by registry observations suggesting that the risk of developing CKD in PA increases with age. ${ }^{2}$ In addition, a recent retrospective case review study of liver transplantation in PA showed that half of patients (4/8) were in stage 2 CKD before the procedure, and $100 \%$ of patients $(4 / 4)$ were in stage $2-3$ CKD in the post-transplant period. $^{8}$ In yet another European series, 2/6 patients developed kidney dysfunction after receiving a liver transplant when their diet was liberalized. ${ }^{9}$ These clinical observations suggest that $\mathrm{CKD}$ is an under-recognized disease-related complication in PA, especially in adults.

Due to the ramifications of a CKD diagnosis for routine monitoring and long-term management, it is important to define the natural history of renal involvement in PA especially because traditional markers of kidney disease, such as increases in blood urea and serum creatinine, may be obscured by the ingestion of a low protein diet and decreased muscle mass, both of which are common in the PA patient population. We have therefore studied a large and genetically heterogeneous cohort of PA patients enrolled in a natural history protocol to approximate the incidence of CKD and identify risk factors associated with

${ }^{1}$ National Human Genome Research Institute, National Institutes of Health, Bethesda, MD, USA; ${ }^{2}$ Nutrition Department, Clinical Research Center, National Institutes of Health,
Bethesda, MD, USA; ${ }^{3}$ National Heart Lung and Blood Institute, National Institutes of Health, Bethesda, MD, USA; ${ }^{4}$ National Institute of Diabetes and Digestive and Kidney Diseases, National Institutes of Health, Bethesda, MD, USA. Correspondence: Charles P. Venditti (venditti@mail.nih.gov) 
progression. Our observations provide important new insights into the manifestations of CKD in PA patients and help establish a framework for the prospective monitoring and the laboratory assessment of renal complications in this population.

\section{MATERIALS AND METHODS Patient cohort and protocols}

The diagnosis of PA was confirmed using a combination of biochemical, clinical, and molecular testing before consent and enrollment into a natural history study (ClinicalTrials.gov identifier: NCT02890342). Study protocol was reviewed and approved by the National Institutes of Health Institutional Review Board (NIH IRB). All subjects or their legal guardians provided written informed consent. Thirty-one individuals with PA, ages 4-53 years, were evaluated over the course of a week-long admission at the NIH Clinical Research Center. Four individuals were of African American descent (4/31, 13\%), 23/31 (74\%) were Caucasian, and 4/31 (13\%) were Hispanic. Clinical data were further enriched using selected results, such as genotype, obtained from outside clinical records, or values from other $\mathrm{NIH}$ protocols (ClinicalTrials. gov identifiers: NCT00369421 and NCT01780168) when subjects were co-enrolled.

\section{Clinical and laboratory studies}

Testing related to renal function included the measurements of blood pressure, serum creatinine, cystatin C, plasma bicarbonate, total serum calcium, phosphorus, 25-OH-vitamin D, 1,25-OH-vitamin D, intact parathyroid hormone (PTH), erythropoietin, and 24-hour creatinine clearance. Imaging included abdominal ultrasound and transthoracic echocardiography. In pediatric patients (ages $<19$ years), eGFR was calculated using the updated creatinine-based Schwartz bedside equation (2009) ${ }^{10}$ and cystatin C-based Schwartz equation (2012). ${ }^{11}$ In patients $>19$ years of age, eGFR was estimated using the Chronic Kidney Disease Epidemiology Collaboration (CKD-EPI) creatinine equation (2009), ${ }^{12}$ and the CKD-EPI cystatin C equation (2012). ${ }^{13}$

\section{Statistics}

Creatinine-based and cystatin C-based methods of estimating GFR were compared either using a paired $t$ test of the means after checking normality with the D'Agostino-Pearson test or using linear regression fitting followed by the slope and intercept comparisons. Kidney growth analysis in pediatric PA patients was performed on the maximal renal length data, using a slope and intercept comparison with a published reference group matched for age and sex. ${ }^{14}$ To compare daily creatinine excretion among PA subjects, age-, height-, and sexadjusted $z$-scores were calculated using the means and standard deviations from published reference populations. ${ }^{15,16}$ A $P$ value $<0.05$ was interpreted as statistically significant. Statistical analysis was performed using GraphPad Prism 6 (GraphPad Software, La Jolla, CA).

\section{RESULTS}

\section{Patient characteristics}

Selected clinical, genetic, and laboratory parameters are summarized in Table 1. Among 31 participants, 55\% were female and $26 \%$ of the participants were older than 19 years of age. Biallelic pathogenic variants were present in PCCA (13 subjects) and PCCB (17 subjects) (Table 1). In one participant, molecular confirmation was not obtained. Three participants $(3 / 31,10 \%)$ had received a liver transplant for metabolic instability at ages 9,13 , and 35 months.

\section{Renal indices}

The mean renal length in PA subjects, irrespective of the underlying renal function estimates, was similar to the published control populations (Supplementary Fig. 1A, B). Three patients $(10 \%)$ had simple renal cysts (Supplementary Fig. 2). In this cross-sectional cohort, the frequency of simple cysts was comparable with the prevalence of simple renal cysts in the general population (10.7\% in Chang et al). ${ }^{17}$ Low serum phosphate was identified in $1 / 31$ (3\%), low 1,25-OH-vitamin $\mathrm{D}$ was present in $2 / 31(6 \%)$, elevated intact $\mathrm{PTH}$ was present $3 / 31(10 \%)$, elevated total serum calcium was present in $3 / 31$ (10\%), low plasma bicarbonate was present in $10 / 31(32 \%)$, and a history of hypertension was present in 6/31 (19\%) (Table 1). Nine of 31 participants were taking $\beta$-blockers for cardiac indications and one participant was also taking an angiotensin II receptor blocker for hypertension, which affected our ability to assess whether hypertension was present at the time of evaluation. Transthoracic echocardiogram revealed reduced left ventricular contractility in $4 / 31$ patients (13\%). Erythropoietin was elevated in $7 / 27$ subjects (26\%) and correlated with bone marrow suppression. Crosssectional analysis of creatinine eGFR in nontransplanted patients suggested an age-dependent pattern of renal function decline (Fig. 1a, $P$ value $<0.002, r=-0.536$ ). However, the cystatin $C$ eGFR in nontransplanted patients revealed that eGFR was reduced in the first decade of life and progressively declined in some subjects in adulthood (Fig. 1b). In pediatric patients, creatinine and cystatin $\mathrm{C}$ eGFRs showed significant discrepancy (Fig. 1c, $P$ value <0.0001). In adults, the prevalence of an eGFR $<60 \mathrm{~mL} / \mathrm{min} / 1.73 \mathrm{~m}^{2}$ was $50 \%(4 / 8)$ by both the CKD-EPI creatinine-based (2009) formula and the CKD-EPI cystatin C equation (2012) (Fig. 1c). However, across all ages and all CKD categories (stages G2-G4), creatinine eGFRs classified only 4/31 (13\%) patients as having CKD, while the cystatin C eGFRs classified 25/30 patients $(82 \%)$ to have an eGFR $<90 \mathrm{~mL} / \mathrm{min} / 1.73 \mathrm{~m}^{2}$ (Fisher's exact test, $P$ value $<0.0001)$. When we compared regression lines of the creatinine eGFR and cystatin $\mathrm{C}$ eGFR in nontransplanted patients (Fig. 1d), the $y$-axis intercept of the creatinine eGFR regression line was significantly higher than the intercept for cystatin C eGFR $(P$ value $<0.0001)$ and the difference between slopes approached statistical significance $(P$ value $=0.0508)$.

We reasoned that sources of discrepancy between the creatinine and cystatin C eGFR in PA patients could be due to lower-than-average serum creatinine concentrations resulting 


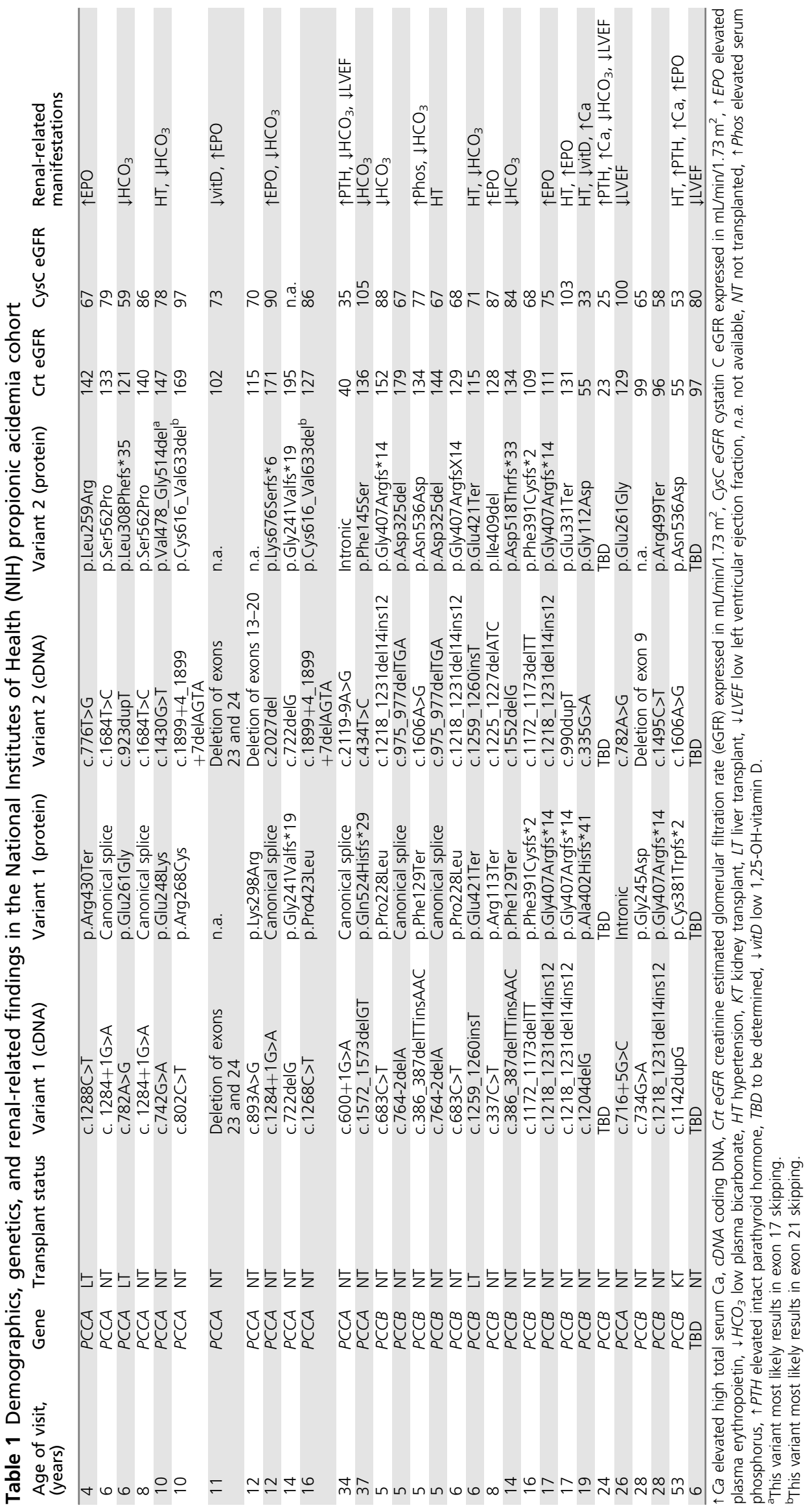


a

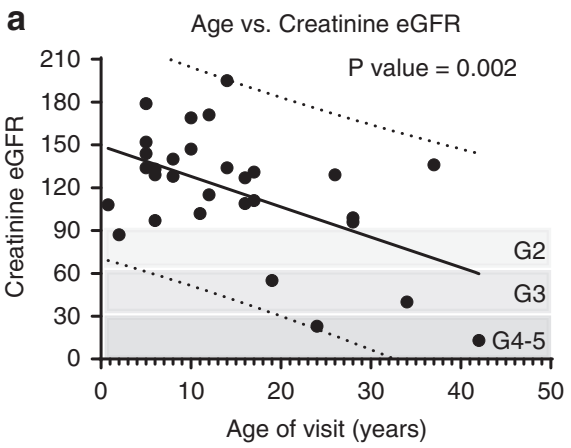

C

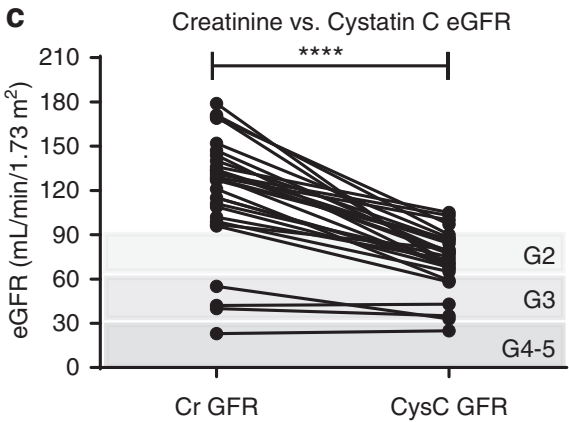

e

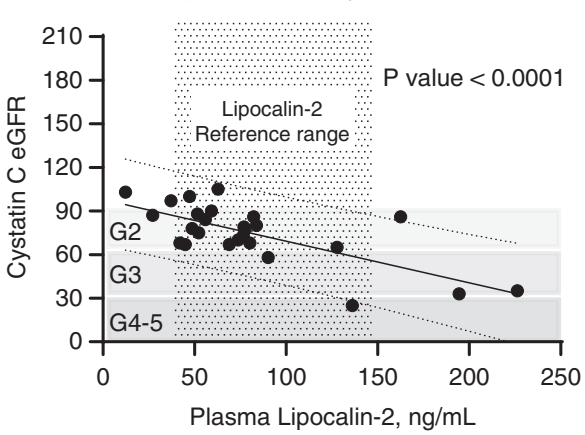

b

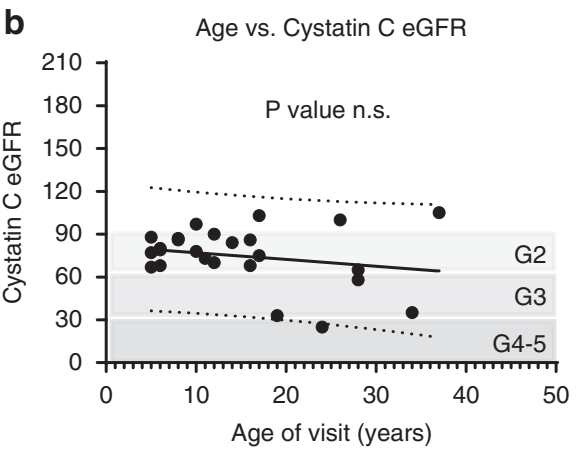

d
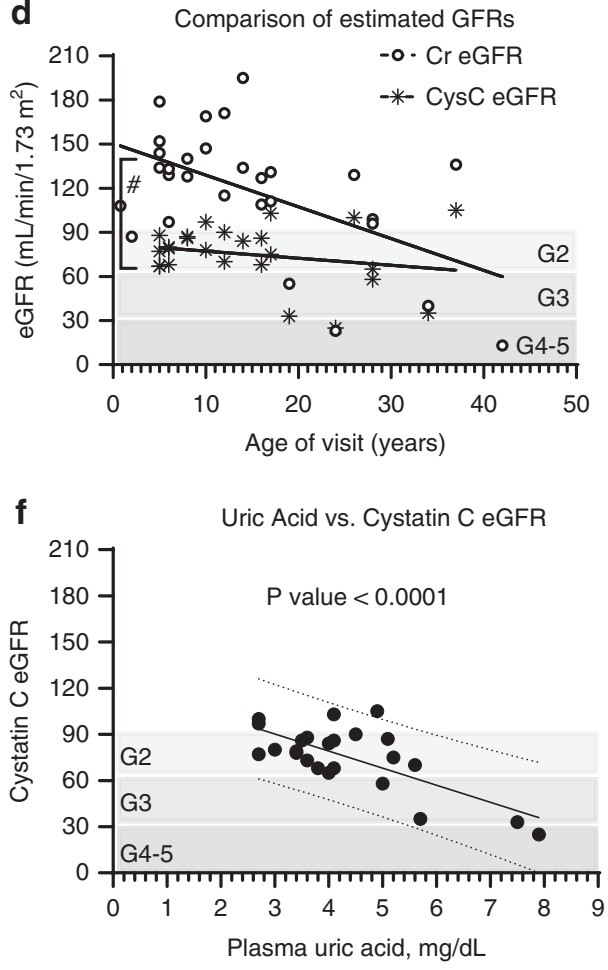

Fig. 1 Significant discrepancy between the creatinine and cystatin C GFR estimates and select laboratory parameters associated with declining estimated glomerular filtration rate (eGFR). a Cross-sectional analysis of creatinine eGFR in nontransplanted patients suggested an agedependent trend of renal function decline ( $P$ value $<0.002, r=-0.536$ ). G2, G3, and G4-5 denote levels of renal function based on eGFR. b Cystatin C eGFR in nontransplanted patients suggested the eGFR was reduced in early childhood, with progressive decline in some subjects in the late teenage or early adult years; $25 / 30$ subjects ( $82 \%$ ) had eGFR $<90 \mathrm{~mL} / \mathrm{min} / 1.73 \mathrm{~m}^{2}$ (chronic kidney disease stages 2-4). c Creatinine (Cr eGFR) and cystatin C eGFRs (CysC eGFR) showed significant disagreement $(P$ value $<0.0001)$. $\mathbf{d}$ Linear regression of creatinine eGFR and cystatin $C$ eGFR in nontransplanted patients demonstrated that the intercept of the creatinine eGFR was significantly different from cystatin C eGFR ( $P$ value $<0.0001$, denoted by the \# sign). e Plasma lipocalin-2, a biomarker of tubular injury, was associated with the cystatin C eGFR ( $P$ value $<0.0001, r=-0.717)$. f Plasma uric acid, a factor that can be associated with chronic kidney disease (CKD), rises with decreasing cystatin C eGFR ( $P$ value $<0.0001, r=-0.718$ ). Dotted lines in $\mathbf{a}, \mathbf{b}, \mathbf{e}, \mathbf{f}$ represent $95 \%$ prediction bands.

from either impaired growth, sarcopenia, meat-restricted diets, reduced methionine intake, diminished synthesis of creatine in the liver, and/or increased tubular secretion of creatinine. Therefore, to explore potential relationships between these variables, we performed linear regression analyses between serum creatinine or cystatin C, using protein consumption, intake of protein equivalent from medical foods, height, body mass index, and body composition measured by whole-body DEXA as independent variables (Supplementary Table 1).

Surprisingly, we found no association between serum creatinine and \% lean body mass or dietary composition and next evaluated daily creatinine excretion adjusted for age, height, and sex. ${ }^{15,16}$ The average adjusted $z$-scores for 24 -hour creatinine excretion were -0.536 in PA girls $(n=6)$ and -0.513 in PA boys $(n=5)$ (Supplementary Table 2). One adult male patient had a daily creatinine excretion $z$-score of -2.518 . In adult female patients, the daily average adjusted $z$-score of creatinine excretion was $-1.622(n=5)$. The finding that PA patients have a below-average creatinine excretion was corroborated by the high prevalence of elevated urinary protein/creatinine ratio (13/21 patients, $62 \%)$. However, only 3 of 18 (17\%) patients showed minimal proteinuria on 24 hour urine protein analyses, and the rest had normal protein 
excretion (Supplementary Fig. 3). Lastly, we performed an unbiased linear regression analysis between creatinine or cystatin $\mathrm{C}$ and other clinical and laboratory parameters, which revealed associations with parameters implicated in mechanisms of renal dysfunction including serum uric acid, prealbumin, measured plasma osmolality, plasma lipocalin 2 , intact parathyroid hormone, total serum calcium, and left ventricular ejection fraction (Supplementary Table 1).

\section{DISCUSSION}

The improved ascertainment and survival of patients with PA over the last several decades mandates recognition of longterm complications. Although recent publications have suggested an association of CKD with propionic acidemia, ${ }^{2,5,7}$ the prevalence of CKD has remained difficult to establish because reported data derive mainly from either isolated case reports, small patient series, and registries. Therefore, we sought to investigate renal disease in a study populated with a relatively large, clinically diverse, and genetically heterogeneous cohort of PA patients that included $\sim 25 \%$ adults.

Using the accepted definition of eGFR $<60 \mathrm{~mL} / \mathrm{min} / 1.73$ $\mathrm{m}^{2}$, we observed an unexpectedly high prevalence of CKD (50\%) among adult PA patients. While this value is lower than the nearly universal prevalence of CKD seen in $m u t^{0}$ methylmalonic acidemia patients, ${ }^{4}$ it is higher than what has been reported $(\sim 15 \%)$ in adult PA patients in the E-IMD registry. ${ }^{2}$ After extending our analyses to younger patients, we identified a significant discrepancy between creatinine and cystatin C GFRs, with the bedside Schwartz creatinine-based equation (2009) resulting in significantly higher GFR estimates compared with those generated using the cystatin $\mathrm{C}-$ based equation. We hypothesized that higher creatinine GFR estimates could be the result of lower serum creatinine levels driven by sarcopenia, diet, or impaired creatine synthesis in the liver. While we did not observe an association between the percent lean body weight and serum creatinine, we found that PA patients had low adjusted daily creatinine excretion. Therefore, we suggest that PA can lead to the reduction of whole-body creatine synthesis, which is likely the result of a combination of dietary and metabolic influences. Several factors inherent to organic acidemias, but known to affect whole-body creatine synthesis, could lead to lower plasma creatinine, and thus bias GFR estimates. These could include dietary restriction of methionine and branched-chain amino acids, reduced intake of meats, low levels of physical activity, and/or impaired energy metabolism in the liver and muscle arising from the mitochondrial dysfunction. ${ }^{18}$

The cellular mechanisms underlying the decline in renal function in propionic acidemia have not been elucidated. While the evaluation of blood and urinary parameters in our study did not reveal a specific pattern that would point to a dominant mechanism(s) of injury (Supplementary Table 1 and Supplementary Fig. 3), our observations are consistent with a single published case report describing nonspecific tubulointerstitial pathology and glomerular sclerosis. ${ }^{7}$ Likewise, the associations between serum creatinine or cystatin $\mathrm{C}$ and plasma lipocalin-2, similar to MMA, ${ }^{19}$ further suggest the role of ongoing tubular injury in the etiology of CKD in PA.

Without direct GFR measurements, this study cannot resolve the question whether cystatin $\mathrm{C}$ eGFR is superior to creatinine eGFR to establish the diagnosis of CKD. Although we did not measure eGFR using iothalamate or iohexol clearance, which, unlike creatinine, are not affected by renal tubular secretion, the constellation of laboratory and imaging studies featured in this paper clearly establish renal insufficiency in many patients. The cross-sectional study design further limits our ability to establish the progression of the renal disease, and a participation bias may exist. Detailed protocol-based evaluations of all patients, irrespective of the clinical suspicion of any organ system involvement, enabled observations of renal complications and associated laboratory parameters. In particular, measurements of cystatin C, plasma uric acid, 25-OH-vitamin D, 1,25-OH-vitamin D, PTH, plasma osmolality, and 24-hour protein excretion in PA subjects suggest their usefulness as part of CKD surveillance.

The late recognition of kidney disease in PA can impede the initiation of renal protective therapies aimed at delaying the onset or slowing the progression of CKD, such as aggressive control of the acid/base status, control of blood pressure through inhibition of the renin-angiotensin system, and urate-lowering therapy. ${ }^{20}$ The diagnosis of CKD might also inform decisions about the optimal timing for liver or combined organ transplantation. Because PA patients have minimal hepatic capacity to metabolize propionic acid, renal excretion is needed to clear "toxic" metabolites, which, by virtue of increased concentrations in the setting of renal insufficiency, might contribute to disease progression in other organ systems. For example, whether worsening kidney disease has cardiac consequences seems possible, given that we observed a strong negative correlation between the left ventricular ejection fraction and renal indices in PA patients with CKD categories G3 and G4.

\section{SUPPLEMENTARY INFORMATION}

The online version of this article (https://doi.org/10.1038/s41436019-0593-z) contains supplementary material, which is available to authorized users.

\section{ACKNOWLEDGEMENTS}

We thank the patients, families, NIH Clinical Center clinical staff, and referring health-care providers for enabling this $\mathrm{NIH}$ natural history study. We thank Julia Fekecs from the National Human Genome Research Institute (NHGRI) for help with medical illustrations and Niraj Triverdi (NHGRI, NIH, Bethesda) for his guidance in designing statistical analysis. We thank Qian (Katie) Sun from the Department of Laboratory Medicine at the NIH Clinical Center for help with the "Materials and Methods" section of the manuscript. This work was supported by the Intramural Research Programs of the National Human Genome Research Institute, the National Institute of Diabetes and Digestive and Kidney Diseases, and the National Heart Lung and Blood Institute. 


\section{DISCLOSURE}

The authors declare no conflicts of interest.

Publisher's note: Springer Nature remains neutral with regard to jurisdictional claims in published maps and institutional affiliations.

\section{REFERENCES}

1. Shchelochkov OA, Carrillo N, Venditti C. Propionic acidemia. In: Adam MP, Ardinger HH, Pagon RAGeneReviews. Seattle, WA: University of Washington; 1993.

2. Kölker $S$, Valayannopoulos $V$, Burlina $A B$, et al. The phenotypic spectrum of organic acidurias and urea cycle disorders. Part 2: the evolving clinical phenotype. J Inherit Metab Dis. 2015;38:1059-1074.

3. Horster F, Baumgartner MR, Viardot $C$, et al. Long-term outcome in methylmalonic acidurias is influenced by the underlying defect (mut0, mut-, cblA, cblB). Pediatr Res. 2007;62:225-230.

4. Kruszka PS, Manoli I, Sloan JL, Kopp JB, Venditti CP. Renal growth in isolated methylmalonic acidemia. Genet Med. 2013;15:990-996.

5. Lam C, Desviat LR, Perez-Cerda C, Ugarte M, Barshop BA, Cederbaum S. 45-Year-old female with propionic acidemia, renal failure, and premature ovarian failure; late complications of propionic acidemia?. Mol Genet Metab. 2011;103:338-340.

6. Lehnert W, Sperl W, Suormala T, Baumgartner ER. Propionic acidaemia: clinical, biochemical and therapeutic aspects. Eur J Pediatr. 1994;153: S68-S80.

7. Vernon HJ, Bagnasco S, Hamosh A, Sperati CJ. Chronic kidney disease in an adult with propionic acidemia. JIMD Rep. 2014;12:5-10.

8. Charbit-Henrion F, Lacaille F, McKiernan P, et al. Early and late complications after liver transplantation for propionic acidemia in children: a two centers study. Am J Transplant. 2015;15:786-791.
9. Quintero J, Molera C, Juamperez J, et al. The role of liver transplantation in propionic acidemia. Liver Transpl. 2018;24:1736-1745.

10. Schwartz GJ, Work DF. Measurement and estimation of GFR in children and adolescents. Clin J Am Soc Nephrol. 2009;4:1832-1843.

11. Schwartz GJ, Schneider MF, Maier PS, et al. Improved equations estimating GFR in children with chronic kidney disease using an immunonephelometric determination of cystatin C. Kidney Int. 2012;82: 445-453.

12. Levey $A S$, Stevens $L A$, Schmid $C H$, et al. A new equation to estimate glomerular filtration rate. Ann Intern Med. 2009;150:604-612.

13. Inker $L A$, Schmid $C H$, Tighiouart $H$, et al. Estimating glomerular filtration rate from serum creatinine and cystatin C. N Engl J Med. 2012;367: 20-29.

14. Rosenbaum DM, Korngold E, Teele RL. Sonographic assessment of renal length in normal children. AJR Am J Roentgenol. 1984;142: 467-469.

15. Johner SA, Boeing $H$, Thamm M, Remer T. Urinary 24-h creatinine excretion in adults and its use as a simple tool for the estimation of daily urinary analyte excretion from analyte/creatinine ratios in populations. Eur J Clin Nutr. 2015;69:1336

16. Remer T, Neubert A, Maser-Gluth C. Anthropometry-based reference values for 24-h urinary creatinine excretion during growth and their use in endocrine and nutritional research. Am J Clin Nutr. 2002;75: 561-569.

17. Chang CC, Kuo JY, Chan WL, Chen KK, Chang LS. Prevalence and clinical characteristics of simple renal cyst. J Chin Med Assoc. 2007;70:486-491.

18. Chandler RJ, Zerfas PM, Shanske S, et al. Mitochondrial dysfunction in mut methylmalonic acidemia. FASEB J. 2009;23:1252-1261.

19. Manoli I, Sysol JR, Li L, et al. Targeting proximal tubule mitochondrial dysfunction attenuates the renal disease of methylmalonic acidemia. Proc Natl Acad Sci USA. 2013;110:13552-13557.

20. Ward F, Holian J, Murray PT. Drug therapies to delay the progression of chronic kidney disease. Clin Med (Lond.). 2015;15:550-557. 